\title{
Phylogenetic position of Oxygyne shinzatoi (Burmanniaceae) inferred from 18S rDNA sequences
}

\author{
Jun Yokoyama $\cdot$ Yayoi Koizumi $\cdot$ Masatsugu Yokota \\ Hirokazu Tsukaya
}

Published online: 18 June 2008

(C) The Botanical Society of Japan and Springer 2008

Erratum to: J Plant Res (2008) 121:27-32
DOI 10.1007/s10265-007-0136-6

In an article published in the Journal of Plant Research (121:27-32), we reported that we had conducted molecular phylogenetic analyses of Burmanniaceae to reveal the phylogenetic position of Oxygyne shinzatoi, a living sample obtained from the second collection series of the species since its discovery. In that article, we neglected to cite the accession number of the 18SrDNA sequence of

Oxygyne shinzatoi. The sequence was released in the DDBJ/EMBL/GenBank international DNA databases under the accession number AB437090. This information was the first report of the molecular sequence of the genus.

The authors cordially express their sincere gratitude to Dr. V. Merckx for pointing out the error.

The online version of the original article can be found under doi:10.1007/s10265-007-0136-6.

\section{J. Yokoyama}

Department of Biology, Faculty of Science,

Yamagata University, Kojirakawa,

Yamagata, Yamagata 990-8560, Japan

\section{Y. Koizumi}

Graduate School of Life Sciences, Tohoku University,

Aoba-ku, Sendai, Miyagi 980-8578, Japan

\section{Yokota}

Laboratory of Ecology and Systematics, Faculty of Science,

University of the Ryukyus,

Nishihara, Okinawa 903-0213, Japan

H. Tsukaya ( $\square)$

Graduate School of Science, University of Tokyo,

7-3-1 Hongo, Bunkyo-ku, Tokyo 113-0033, Japan

e-mail: tsukaya@nibb.ac.jp

H. Tsukaya

National Institute for Basic Biology, National Institutes

of Natural Sciences, Myodaiji-cho, Okazaki,

Aichi 444-8585, Japan 